\title{
Subspecialty pediatric neurosurgery training: a skill-based training model for neurosurgeons in low-resourced health systems
}

\author{
Michael C. Dewan, MD, MSCl,,${ }^{1,2}$ Justin Onen, MD, ${ }^{3}$ Hansen Bow, MD, PhD, ${ }^{1}$ Peter Ssenyonga, MD, ${ }^{3}$ \\ Charles Howard, MA, MSW, ${ }^{3}$ and Benjamin C. Warf, MD ${ }^{2-4}$ \\ ${ }^{1}$ Department of Neurological Surgery, Vanderbilt University Medical Center, Nashville, Tennessee; ${ }^{2}$ Global Neurosurgery \\ Initiative, Program in Global Surgery and Social Change, Department of Global Health and Social Medicine, Harvard Medical \\ School, Boston, Massachusetts; ${ }^{3}$ CURE Children's Hospital of Uganda, Mbale, Uganda; and ${ }^{4}$ Department of Neurological \\ Surgery, Boston Children's Hospital, Harvard Medical School, Boston, Massachusetts
}

There is inadequate pediatric neurosurgical training to meet the growing burden of disease in low- and middle-income countries (LMIC). Subspecialty expertise in the management of hydrocephalus and spina bifida-two of the most common pediatric neurosurgical conditions_offers a high-yield opportunity to mitigate morbidity and avoid unnecessary death. The CURE Hydrocephalus and Spina Bifida (CHSB) fellowship offers an intensive subspecialty training program designed to equip surgeons from LMIC with the state-of-the-art surgical skills and equipment to most effectively manage common neurosurgical conditions of childhood. Prospective fellows and their home institution undergo a comprehensive evaluation before being accepted for the 8-week training period held at CURE Children's Hospital of Uganda (CCHU) in Mbale, Uganda. The fellowship combines anatomy review, treatment paradigms, a flexible endoscopic simulation $\mathrm{lab}$, daily ward and ICU rounds, radiology rounds, and clinic exposure. The cornerstone of the fellowship is the unique operative experience that includes a high volume of endoscopic third ventriculostomy with choroid plexus cauterization, myelomeningocele closure, and ventriculoperitoneal shunting, among many other procedures performed at $\mathrm{CCHU}$. Upon completion, fellows return to their home institution to establish or rejuvenate a robust pediatric practice as part of a worldwide network of CHSB trainees committed to the care of underserved children. To date, the fellowship has graduated 33 surgeons from 20 different LMIC who are independently performing thousands of hydrocephalus and spina bifida operations each year.

https://thejns.org/doi/abs/10.3171/2018.7.FOCUS18249

KEYWORDS endoscopic third ventriculostomy with choroid plexus cauterization; hydrocephalus; pediatric neurosurgery; spina bifida; training

$\mathrm{T}$ HERE exists a tremendous burden of pediatric neurosurgical disease in low- and middle-income countries (LMIC) ${ }^{2,3}$ Scarce human resources, including a dearth of capable and accessible pediatric neurosurgeons, limit the delivery of life-saving surgical care..$^{10,11}$ LMIC need more neurosurgeons trained and equipped to treat their country's children and thereby avert unnecessary disability and death. ${ }^{13,15}$ These surgeons would ideally be skilled, adequately equipped, familiar with their practice environment and population, and committed to a longterm investment in the community. A transient presence leads to poor follow-up, gaps in surgical coverage, and ultimately distrust among the community being served. ${ }^{4}$ A fundamental component of surgical capacity building is training-training new neurosurgeons as well as procedure-based training to expand or enhance the skill set

ABBREVIATIONS CCHU = CURE Children's Hospital of Uganda; CHSB = CURE Hydrocephalus and Spina Bifida; ETV/CPC = endoscopic third ventriculostomy with choroid plexus cauterization; LMIC = low- and middle-income countries; MM = myelomeningocele; PTL = partner treatment location; REDCap = Research Electronic Data Capture; VPS = ventriculoperitoneal shunting

SUBMITTED May 30, 2018. ACCEPTED July 3, 2018.

INCLUDE WHEN CITING DOI: 10.3171/2018.7.FOCUS18249. 
of existing neurosurgeons. ${ }^{1,5}$ Supplementing a basic skill set with state-of-the-art techniques translates into superior care and improved patient outcomes. ${ }^{12}$ Here we describe a novel, skill-based training paradigm that expands the global delivery of prevailing techniques for neurosurgical care to children with hydrocephalus.

\section{Rationale for Subspecialty Training in Hydrocephalus and Spina Bifida}

Hydrocephalus is one of the most common pathologies encountered by neurosurgeons, and is known to be more common in LMIC. ${ }^{3,9}$ Two of the most common single causes of infant hydrocephalus in LMIC are neonatal infection (postinfectious hydrocephalus) and myelomeningocele (MM) with the Chiari II malformation. Early surgical treatment of both hydrocephalus and MM (spina bifida) reduces childhood morbidity and saves lives. ${ }^{6}$ The treatment for hydrocephalus has become more complex as new treatment modalities beyond shunt placement have emerged. ${ }^{7,14}$ Whereas experience with alternative endoscopic treatments for hydrocephalus is now a compulsory part of many modern training programs, trainees in resource-poor settings often are not exposed to the same level and breadth of training. ${ }^{4}$ Equipping surgeons worldwide with endoscopic approaches to CSF diversion and reduction-which may ultimately reduce readmissions, reoperations, costs, and mortality - will build surgical capacity and more effectively address the deficit in neurosurgical care. ${ }^{16}$ Such training is particularly important for surgeons in LMIC, where shunt complications are more likely to result in severe neurological injury or death because of barriers to obtaining emergency neurosurgical treatment.

Endoscopic third ventriculostomy with choroid plexus cauterization (ETV/CPC) is a specific innovation that can avoid many of the complications related to shunt insertion and dependence, without compromising neurocognitive development. ${ }^{8}$ Unlike shunt malfunctions that can occur at any time over the patient's life, the great majority of ETV/ CPC treatment failures occur within the first 6 months after surgery. For infants, this translates into a "safe zone" during which failure is visible to the caregiver (e.g., bulging fontanel and rapid head growth) and is typically not an emergency, providing time for the caregiver to return for intervention. Beyond the 6-month postoperative window, ETV/CPC success is durable and avoids the lifelong risk of shunt malfunction. Accordingly, expanding the workforce of LMIC neurosurgeons proficient with ETV/CPC stands to dramatically improve long-term outcomes for children with hydrocephalus in these challenging environments.

As the field of neurosurgery seeks ways to reduce inequities in the delivery of neurosurgical care, the CURE Hydrocephalus and Spina Bifida (CHSB) fellowship represents a high-yield training paradigm designed to impact a large population by expanding access to ETV/CPC as well as to sound methods of managing shunts, and also to the optimal initial and subsequent management of infants born with MM. It is our hope that the achievements made, barriers encountered, and lessons learned from this model can guide the development of other subspecialty training initiatives in resource-poor settings.

\section{General Model}

The CHSB training program at CURE Children's Hospital of Uganda (CCHU) is an intensive 8-week clinical immersion whereby trainees study comprehensive management of hydrocephalus and spina bifida, from diagnosis and workup to surgical management and postoperative follow-up (Fig. 1). There is a particular focus on mastering ETV/CPC, but the training also includes shunt placement and management of complications, initial closure of MM, and longer-term management of children with MM. Upon completion of the program, trainees return to their home institution as partners in a global network of pediatric neurosurgeons to implement the knowledge and skills gained for the benefit of children in their home country. CHSB ensures that the trainee has access to the necessary equipment and also provides an on-site clinical care coordinator to assist with patient follow-up and data collection.

\section{Identification, Recruitment, and Funding of CHSB Fellows}

Eligible fellows are those who have completed standard neurosurgical training as defined by their country's Ministry of Health, surgical accreditation governing body, or equivalent thereof. The ideal prospective fellow resides in a resource-limited setting that observes a high volume of underserved patients with hydrocephalus and spinal dysraphism. Occasionally, fellows are identified by word of mouth via the transnational interpersonal relationships that have been established by the current neurosurgeons and administration at CCHU. Additionally, CHSB solicits the application of interested trainees via its website (https://cure.org/hydrocephalus/), which generates between 10 and 15 qualified applicants annually who are considered for the fellowship.

Before acceptance to the fellowship, there is a rigorous screening of both the fellow and the associated institution to confirm three essential features that CHSB considers requisite for long-term success. First, there must be sufficient clinical volume to justify the costs of the training and required equipment, as well as to maintain competency. Second, there must be institutional/hospital support to facilitate the fellow's pediatric neurosurgical practice upon return. Third, there must be a commitment from both the fellow and the institution/hospital to participate in collaborative data collection for the purpose of improving treatment and outcomes. Before a prospective trainee is admitted to the fellowship program, a representative of CHSB will personally visit the trainee's home institution to ensure that these criteria are met.

Financial support of the fellows derives both from scholarship support via CHSB and directly from the institution or department from which the fellow originates. The shared approach cultivates ownership of the fellow's success and trajectory by both parties. The fellowship cost for each trainee, including travel, medical license, per diem expenses, lodging, and faculty time and equipment use, is roughly USD $\$ 15,000$. CHSB works with each fellow's home institution to ensure sufficient access to the necessary video-endoscopy equipment. Within the year before and after the fellowship, an additional USD 


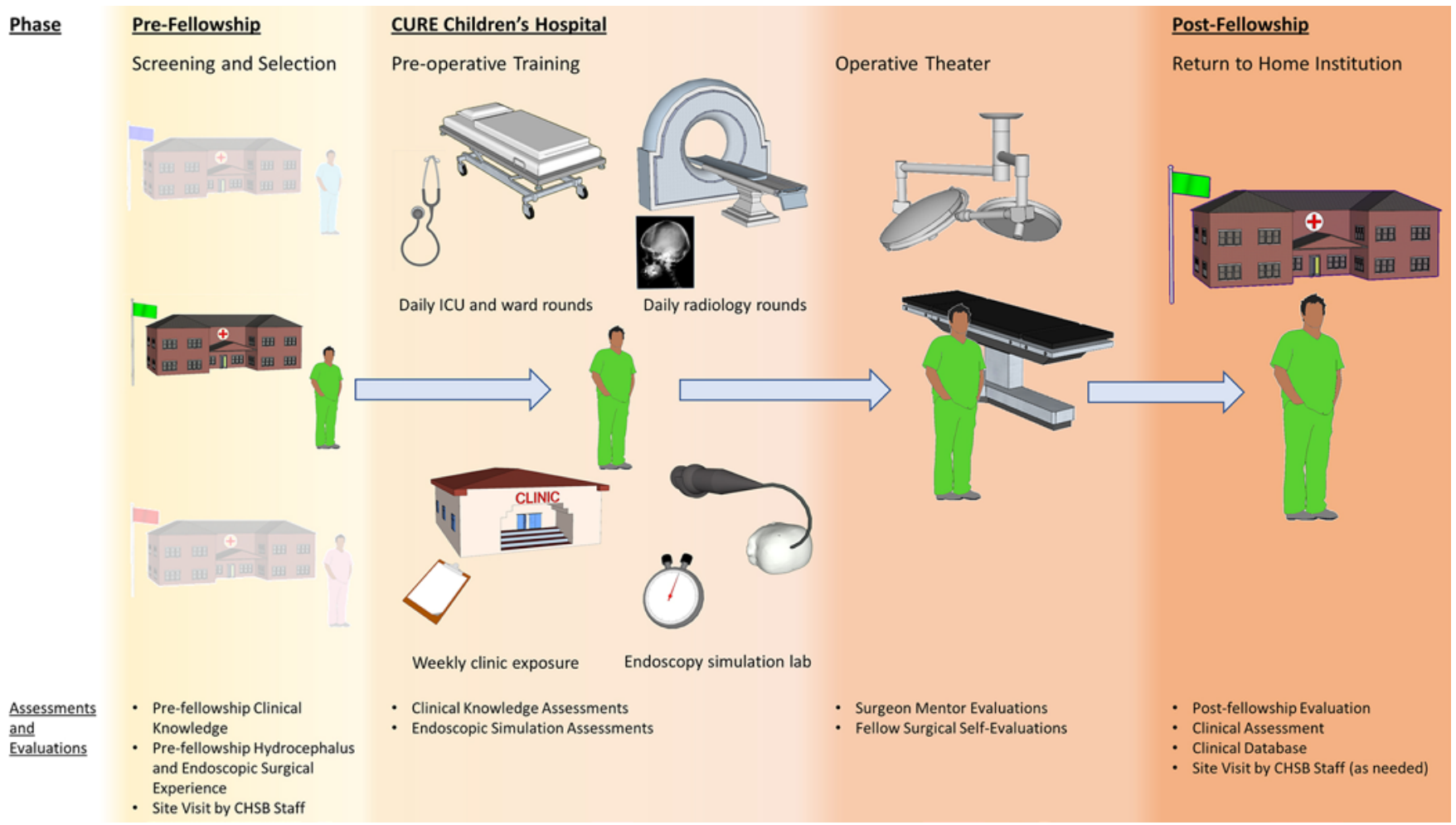

FIG. 1. CHSB training model flow diagram. Prospective surgeons undergo detailed screening to ensure compatibility and likelihood of long-term success. During the 8-week experience, bedside teaching and intensive operative training are emphasized to deliver a comprehensive expertise in the prevailing, multimodal management of hydrocephalus and spina bifida. Throughout, fellows are evaluated by CHSB faculty and via self-evaluations. After the fellowship, graduates return to their home institution, maintaining a partnership with CHSB via continued evaluations and participation in a clinical research database. Models in this figure were created by SketchUp (Trimble Inc.) using components from 3D Warehouse.

$\$ 20,000$ is budgeted to cover the costs of trainee selection and vetting (prefellowship), care coordinator salary, and postfellowship site visits by CHSB staff.

\section{Fellowship Experience}

\section{Prearrival}

Trainees are sent an orientation packet with information about Uganda and the training hospital (CCHU), travel details, daily schedules, cultural norms, and general tips for a successful experience. Included in the packet is a procedure manual for the combined ETV/CPC, which not only details indications, anatomy, and technique, but also outlines important treatment algorithms for hydrocephalus management in LMIC. Relevant literature on hydrocephalus and spina bifida management is also provided. Fellows are expected to review all materials in detail before their arrival to CCHU. A brief web-based knowledge and experience assessment is completed by the trainee before the first day of fellowship, granting CHSB staff an understanding of the fellow's current competency.

\section{Clinical Activities \\ Simulation}

ETV/CPC is performed using a flexible endoscope - an instrument foreign to most neurosurgeons. Thus, learning the procedure first requires comfort with the unique ma- neuverability and control of this instrument. On their first day of the fellowship, trainees are oriented to an endoscopic simulation station outfitted with cranial models approximating the size and intracranial contents of various infants with hydrocephalus (Fig. 2). Each cranial model, designed from thin-cut radiographs, is 3D printed at a cost of roughly USD $\$ 4$. In this simulation, the fellow learns to position the patient's head, set up the camera, position the endoscope, and utilize the working channel and accompanying accessories. Most importantly, the fellow becomes comfortable with the twists and turns of the flexible endoscope, while navigating the intraventricular space in a safe and leisurely environment. Eventually, the fellow is timed while he or she steers the scope from one predefined anatomical position to the next, incurring a time penalty for each inappropriate maneuver. After becoming familiar with the flexible endoscope, training shifts to the operating theater.

\section{Operative Experience}

The unparalleled operative experience at $\mathrm{CCHU}$ represents the highlight of the fellowship. During the 8-week experience, fellows perform roughly 50 endoscopic cases, 25 shunt cases, and 20 procedures for spinal dysraphism. Depending on the interest of and progression by the fellow through the curriculum, he or she may participate in the surgical management of other pathologies commonly seen 


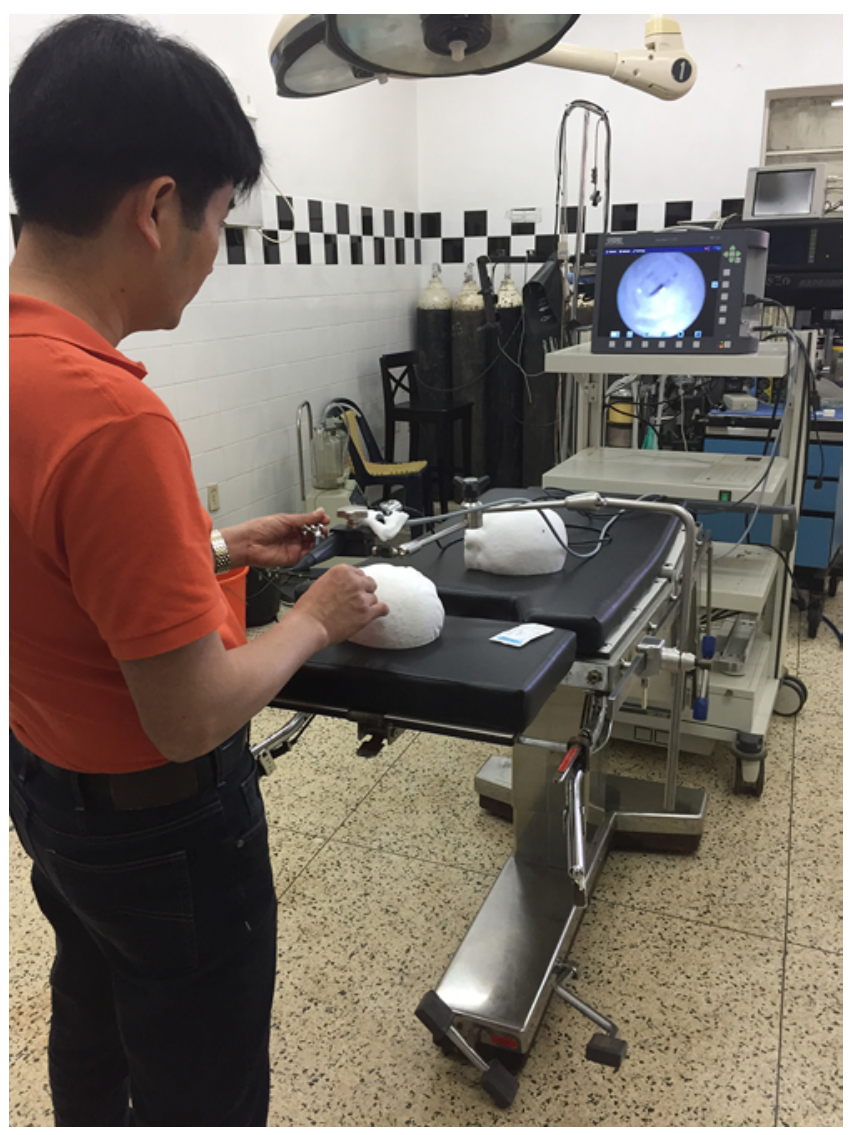

FIG. 2. Endoscopic simulation. A CHSB fellow practices using the flexible endoscope in the simulation laboratory. Cranial models are 3D printed to simulate the intraventricular anatomy of a variety of patients with hydrocephalus.

at CCHU, including tumors, trauma, and CNS infections. The focus of the fellowship, however, resides with hydrocephalus and spina bifida management.

Two full-time neurosurgeons at CCHU-both Ugandans with subspecialty training in endoscopy-provide direct oversight and instruction in the operating theater (Fig. 3 ). As with any surgical training program, there is a graduated level of responsibility. As basic concepts are demonstrated, trainees are introduced to more complex patients and more complex maneuvers. By the final week, fellows independently perform all surgeries for hydrocephalus (ETV/CPC, ventriculoperitoneal shunting [VPS]) and spinal dysraphism (MM closure, encephalocele repair) with only distant oversight.

\section{Daily Rounds}

Teaching rounds are led by the medical director each morning in the ICU and on the ward. The fellow participates in treatment plans made by the team of physicians and nurses regarding new patients admitted overnight, those going to the operating theater, and any patients recovering from recent surgery.

Each afternoon, the team of surgeons meets with the house staff to discuss new inpatients and those seen that day in clinic with pathology that will potentially require

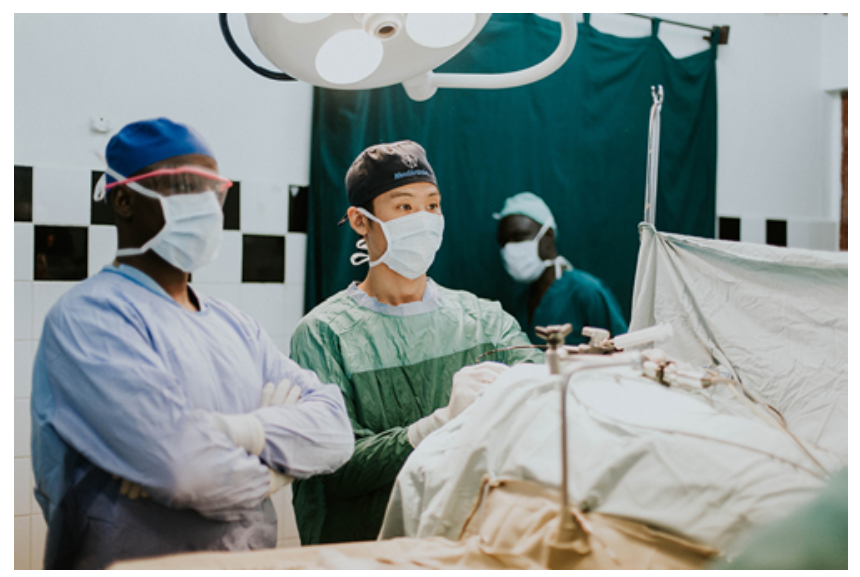

FIG. 3. Operative experience. CHSB faculty (left) provides teaching and oversight as a trainee (center) gains experience with ETVICPC.

an operation. Here, the fellow learns about surgical decision-making, including whether to offer ETV or VPS, or to sample CSF and observe. Any imaging obtained from the on-site CT scanner is also reviewed and treatment plans are discussed. An environment of academic curiosity and clinical inquisitiveness by the fellow is promoted.

\section{Clinic}

On days during which the operative caseload is light, fellows are encouraged to attend clinic, which is organized and facilitated by senior house staff and overseen by the consultant neurosurgeons. The hospital accepts and evaluates patients every day of the week. On a rotating basis, satellite clinics are held every month in distant regions of the country to expand access to pediatric neurosurgical care and to facilitate postoperative follow-up. Fellows are encouraged to participate in these off-site clinics. Finding this patient-centered means of follow-up to be useful, some graduates have imitated this model in their home country.

\section{Clinical Practice and Research Collaboration}

The capstone of the training program is the return of the fellow to his or her home institution to establish a robust clinical practice delivering state-of-the-art treatment to an underserved population. Each graduate is provided with two Karl Storz 3.7-mm flexible, steerable ventriculoscopes; an all-in-one telemonitor; and endoscope accessories. Shunt implants are made available via a partnership maintained with the International Federation for Spina Bifida and Hydrocephalus. The combination of the surgeon graduate and his or her home department or institution represents a partner treatment location (PTL) within the CHSB network.

Central to the clinical training is an introduction to scientific discovery. Although formal research is not a requirement, fellowship graduates are given access to a prebuilt data collection platform, Research Electronic Data Capture (REDCap), to organize clinical data and monitor surgical outcomes of patients with hydrocephalus or spina bifida. During the fellowship experience at $\mathrm{CCHU}$, the curriculum includes systematic documentation of patient 
and operative details within the electronic data management software. The mobile software is intended for use by the fellow upon graduation at his or her home institution as a quality-control measure and, if interested, as a resource for scientific exploration. It is notable that funding for the fellowship and equipment includes a full-time care coordinator in-country assigned to the surgeon for the purposes of patient follow-up and data collection. Close clinical follow-up is focused on the first 6 months, because this is the time period during which the great majority of ETV/CPC failures will occur. The designated care coordinator for each surgeon is also trained to use REDCap. Resources are made available to fellowship graduates to analyze their own clinical data for the purposes of scientific presentation and quality improvement if they so choose. This model instills a sense of ownership and pride in one's practice and surgical outcomes.

\section{Preliminary Measures of Success \\ Number and Locations of Fellowship Graduates}

To date, 33 surgeons from 20 LMIC have graduated from the CHSB fellowship program. Current fellowship graduates practice neurosurgery in Angola, Bangladesh, Democratic Republic of Congo, Ethiopia, Ghana, Guinea, Honduras, Indonesia, Kenya, Liberia, Malawi, Mali, Mozambique, Nepal, Nigeria, Philippines, Tanzania, Uganda, Vietnam, and Zambia. In association with past fellows, CHSB maintains 17 PTLs throughout Africa and Southeast Asia. In addition, 12 neurosurgeons from North America and Europe have also undergone similar training at CCHU and, in turn, have trained other surgeons in resource-limited settings.

\section{Types and Numbers of Cases Performed}

Since 2014, CHSB has tracked basic hydrocephalus case volume figures of graduates at all PTLs with which we have a research collaborative. During the first year in which records were obtained, 1091 cases were performed across 9 PTLs, including 388 ETV and 565 VPS procedures. Twenty percent of VPS procedures were a revision of a prior shunt insertion, whereas $6 \%$ of ETV procedures were a revision of a failed ETV. By 2017, the number of PTLs had nearly doubled to 17 sites, with more than 2500 hydrocephalus operations performed over the calendar year, including 963 ETV and 1582 shunt insertions or shunt revisions. Across the entire CHSB network (including CCHU), nearly half (48\%) of all patients who present with newly diagnosed hydrocephalus from any cause are offered a shunt-free treatment option in the form of an ETV/CPC or ETV alone. This represents a dramatic shift from pre-CHSB years, wherein nearly $100 \%$ of children with hydrocephalus who presented to these hospitals were offered VPS only.

Myelomeningocele closures and other dysraphism procedures have been recorded since 2017 only, when CURE Hydrocephalus expanded its scope to become CHSB; 405 such procedures were performed across the 17 PTLs.

\section{Academic Productivity}

Beyond offering clinical care to their population, many graduates of the training program have exercised scientific curiosity and shared their experiences in the neurosurgical literature. During their 8-week fellowship, trainees are exposed to the current literature and encouraged to explore clinical questions. Their instruction in and familiarization with the utility of REDCap incites hypothesis generation and data exploration. As of May 2018, 11 graduates from LMIC have authored 55 MEDLINE-indexed manuscripts since their fellowship training, of which 21 manuscripts specifically relate to the management of hydrocephalus and spina bifida. These manuscripts have been published in a variety of journals, including the Journal of Neurosurgery, World Neurosurgery, the New England Journal of Medicine, and The Lancet.

\section{Advantages to the CHSB Model}

Several aspects of the CHSB training program have contributed to its success. First, its setting within a lowincome country more closely approximates the permanent working environment and patient populations of most fellows. Basic perioperative services, modest operating room equipment, limited disposable supplies, and intermittent power outages are just some of the challenges a trainee encounters while on-site. These are the same challenges graduates might expect to find in their home institutions, thus making them better prepared. Second, the extraordinary volume of patients with hydrocephalus and spina bifida treated at CCHU allows trainees an ample number of cases within the relatively short, 8-week fellowship. The result is an efficient, high-yield operative experience, saving time and money for the trainee. Third, the full-time neurosurgical faculty at CCHU are expert surgeons and skilled educators, as should be present in any surgical training hospital. Implicit in their job description is the training of CHSB fellows, who rotate through the hospital on a regular basis. In addition, CHSB employs Ugandan staff members who provide continued support to graduates, including coordinating equipment repair, negotiating with hospital or departmental administration, and building research infrastructure. Last, the CHSB model immediately plugs graduates into a worldwide network of surgeons committed to the care of children with hydrocephalus and spina bifida. Some fellowship alumni have already returned home to become neurosurgical leaders and educators in their own country.

\section{Limitations, Challenges, and Obstacles}

Despite the many advantages to the CHSB model, several important limitations must be considered by any reader considering emulating this capacity-building model. First - and most importantly - the CHSB model of training is not self-sustainable financially. CHSB is funded by a variety of donors, foundations, grants, and partnerships. Beyond simply the direct costs associated with the 8-week fellowship, one must also consider the opportunity costs that accrue to a surgeon who is on leave from his or her home neurosurgical department. Additionally, the CHSB fellowship is not a comprehensive neurosurgical training program; applicants must have completed general training to be considered. Finally, in its current form, the annual 
supply of graduates from $\mathrm{CCHU}$ is woefully inadequate relative to the clinical demand globally. In a calendar year, CHSB can feasibly graduate only 6 surgeons from the program. We are exploring an expansion of the CHSB model to other hospitals in sub-Saharan Africa and in Southeast Asia, which will boost our training capacity dramatically and more efficiently combat the burden of untreated neurosurgical disease among children in LMIC.

\section{Future Directions and Implications for Neurosurgical Capacity Building}

Herein, we offer a detailed description of a neurosurgical skill-based, subspecialty training model for neurosurgeons in LMIC. Focusing on two of the most common neurosurgical conditions, hydrocephalus and spina bifida, the training program seeks to deliver a high-yield experience designed to impact the greatest number of pediatric neurosurgical patients in resource-poor settings. Nonetheless, the unmet neurosurgical burden extends far beyond pediatric hydrocephalus and spina bifida. Training surgeons in LMIC to perform the prevailing techniques for tumor resection, spinal fixation, functional procedures, and many other operations is essential if we are to approach parity in the global delivery of neurosurgical care. The model we describe - considering both its strengths and limitationsmight serve as a rubric for others interested in replicating this program for other pathologies. Moreover, the presence of our graduates in hospitals throughout sub-Saharan Africa and Southeast Asia serves as an excellent avenue by which interested neurosurgeons from high-income countries can develop partnerships and offer mutual mentorship.

\section{References}

1. Dempsey KE, Qureshi MM, Ondoma SM, Dempsey RJ: Effect of geopolitical forces on neurosurgical training in SubSaharan Africa. World Neurosurg 101:196-202, 2017

2. Dewan MC, Rattani A, Fieggen G, Arraez MA, Servadei F, Boop FA, et al: Global neurosurgery: the current capacity and deficit in the provision of essential neurosurgical care. J Neurosurg [epub ahead of print April 27, 2018; DOI: 10.3171/2017.11.JNS171500]

3. Dewan MC, Rattani A, Mekary R, Glancz LJ, Yunusa I, Baticulon RE, et al: Global hydrocephalus epidemiology and incidence: systematic review and meta-analysis. J Neurosurg 37:1-15, 2018

4. Figaji A, Taylor A, Mahmud MR, Bello S, Wegoye E, Ssenyonga $\mathrm{P}$, et al: On progress in Africa, by African experts. Lancet Neurol 17:114, 2018

5. Haglund MM, Kiryabwire J, Parker S, Zomorodi A, MacLeod D, Schroeder R, et al: Surgical capacity building in Uganda through twinning, technology, and training camps. World J Surg 35:1175-1182, 2011

6. Kahle KT, Kulkarni AV, Limbrick DD Jr, Warf BC: Hydrocephalus in children. Lancet 387:788-799, 2016
7. Kulkarni AV, Riva-Cambrin J, Browd SR, Drake JM, Holubkov R, Kestle JRW, et al: Endoscopic third ventriculostomy and choroid plexus cauterization in infants with hydrocephalus: a retrospective Hydrocephalus Clinical Research Network study. J Neurosurg Pediatr 14:224-229, 2014

8. Kulkarni AV, Schiff SJ, Mbabazi-Kabachelor E, Mugamba J, Ssenyonga P, Donnelly R, et al: Endoscopic treatment versus shunting for infant hydrocephalus in Uganda. N Engl J Med 377:2456-2464, 2017

9. Muir RT, Wang S, Warf BC: Global surgery for pediatric hydrocephalus in the developing world: a review of the history, challenges, and future directions. Neurosurg Focus 41(5):E11, 2016

10. Park KB, Johnson WD, Dempsey RJ: Global neurosurgery: the unmet need. World Neurosurg 88:32-35, 2016

11. Punchak M, Mukhopadhyay S, Sachdev S, Hung Y-C, Peeters S, Rattani A, et al: Neurosurgical care: availability and access in low-income and middle-income countries. World Neurosurg 112:e240-e254, 2018

12. Ravindra VM, Kraus KL, Riva-Cambrin JK, Kestle JR: The need for cost-effective neurosurgical innovation-a global surgery initiative. World Neurosurg 84:1458-1461, 2015

13. Rudolfson N, Dewan MC, Park KB, Shrime MG, Meara JG, Alkire BC: The economic consequences of neurosurgical disease in low- and middle-income countries. J Neurosurg $\mathbf{5 4 : 1 - 8 , 2 0 1 8}$

14. Warf BC: Comparison of endoscopic third ventriculostomy alone and combined with choroid plexus cauterization in infants younger than 1 year of age: a prospective study in 550 African children. J Neurosurg 103 (6 Suppl):475-481, 2005

15. Warf BC: "Who is my neighbor?" Global neurosurgery in a non-zero-sum world. World Neurosurg 84:1547-1549, 2015

16. Warf BC, Alkire BC, Bhai S, Hughes C, Schiff SJ, Vincent JR, et al: Costs and benefits of neurosurgical intervention for infant hydrocephalus in sub-Saharan Africa. J Neurosurg Pediatr 8:509-521, 2011

\section{Disclosures}

The authors report no conflict of interest concerning the materials or methods used in this study or the findings specified in this paper.

\section{Author Contributions}

Conception and design: Dewan, Warf. Acquisition of data: Dewan, Howard. Analysis and interpretation of data: Dewan, Howard. Drafting the article: Dewan, Warf, Bow. Critically revising the article: Warf, Dewan, Onen, Bow, Howard. Reviewed submitted version of manuscript: Warf, Dewan, Onen, Ssenyonga, Howard. Approved the final version of the manuscript on behalf of all authors: Warf. Administrative/technical/material support: Warf, Onen, Bow, Ssenyonga, Howard. Study supervision: Warf.

\section{Correspondence}

Benjamin C. Warf: Boston Children's Hospital, Boston, MA. benjamin.warf@childrens.harvard.edu. 